\title{
Retrieving text inferences: Controlled and automatic influences
}

\author{
MURRAY SINGER and GILBERT REMILLARD \\ University of Manitoba, Winnipeg, Manitoba, Canada
}

\begin{abstract}
Bridging inferences contribute to text coherence by identifying the connections among ideas, whereas elaborative inferences simply specify sensible extrapolations from text. Bridging inferences have been indistinguishable from explicit text ideas on numerous measures, suggesting similar longterm memory (LTM) representations for the two, whereas elaborative inferences are inferior. To evaluate the LTM representations of text ideas, we used the extended process-dissociation procedure (Buchner, Erdfelder, \& Vaterrodt-Plunnecke, 1995; Jacoby, 1991) to partition the controlled, recollective contributions to text retrieval from the automatic, familiarity-based contributions. The automatic contribution to the recognition of implied concepts was consistently negligible, an outcome consistent with the absence of perceptual processing of those concepts during the original reading. In addition, the controlled basis of recognition was consistently higher for explicit than for implicit concepts, which suggests a more robust conceptual representation for explicit text ideas (Yonelinas, 2002). These results were interpreted to reflect the asymmetric representation of explicit ideas and inferences (elaborative and even bridging inferences) in the surface, propositional textbase, and situational levels of text representation.
\end{abstract}

In the study of the inferences that contribute to text comprehension, the distinction between bridging and elaborative inferences has been an important one. Bridging inferences identify unstated text connections, and so preserve message coherence. For example, a full understanding of sequence (1) depends on the bridging inference that the events of the second sentence were motivated by the ideas of the first sentence.

(1) Valerie left early for the birthday party. She spent an hour shopping at the mall.

In detecting this relation, the understander accesses pertinent world knowledge, such as the concept PRESENT, and integrates it with the text representation (Singer \& Halldorson, 1996; Singer, Halldorson, Lear, \& Andrusiak, 1992). Considerable evidence has established that bridging inferences are indistinguishable or hardly distinguishable from explicit text ideas on behavioral measures, including inference answer time and the time to name, recognize, and make lexical decisions about implied text concepts (Black \& Bern, 1981; Bloom, Fletcher, van den Broek, Reitz, \& Shapiro, 1990; Keenan, Baillet,

This research was supported by Discovery Grant OGP9800 from the Natural Sciences and Engineering Research Council of Canada (NSERC) to M.S. and graduate fellowships from NSERC and the University of Manitoba to G.R. We thank Karen McClure and Paula MacPherson for their conscientious work in collecting the data for these experiments. We are grateful to Lee Osterhout, Ed O'Brien, and an anonymous reviewer for their guidance in the refinement of this article. Correspondence should be addressed to M. Singer, Department of Psychology, University of Manitoba, Winnipeg, MB, R3T 2N2 Canada (e-mail m_singer@umanitoba.ca).
\& Brown, 1984; Myers, Shinjo, \& Duffy, 1987; Potts, Keenan, \& Golding, 1988; Singer, 1980; Singer \& Ferreira, 1983).

In contrast, elaborative inferences extrapolate from text in sensible ways, but do not play a special role in coherence. In this regard, suppose one reads only the sentence Valerie left early for the birthday party. The inference that Valerie was bringing a present to the party would be sound, but it would not link the sentence to a specific text antecedent. Congruent with this analysis, elaborative inferences are inferior to explicit text ideas on a variety of behavioral measures (Corbett \& Dosher, 1978; McKoon \& Ratcliff, 1986; Potts et al., 1988; Singer, 1980; Singer \& Ferreira, 1983). However, elaborative inferences may be at least transiently activated during comprehension (Keefe \& McDaniel, 1993). Furthermore, elaborative inferences may be encoded in the text representation when they are highly constrained. For example, bird for Thanksgiving constrains the category instantiation turkey (O'Brien, Shank, Myers, \& Rayner, 1988; see also Lucas, Tanenhaus, \& Carlson, 1990; McKoon \& Ratcliff, 1988).

A central concern in this realm has been the long-term memory (LTM) status of the inferences that result from comprehension (e.g., Cook, Limber, \& O'Brien, 2001; Klin, Guzman, \& Levine, 1999; Singer, 1994). Behavioral equivalence of explicit and implicit text ideas has frequently been interpreted to signify the equivalence of their LTM representations. However, there are several reasons to be cautious about this assumption. For example, behavioral equivalence might reflect the aforementioned transient activation of an implied concept during comprehension, rather than its encoding in LTM (Corbett \& Dosher, 1978; Keefe \& McDaniel, 1993; Kintsch, 
1988). Alternatively, special test strategies may influence the respondent to access only a subset among the surface, propositional, and situational levels of representation that result from text comprehension (Schmalhofer \& Glavanov, 1986; van Dijk \& Kintsch, 1983). For example, judging the plausibility of the test probe (Reder, 1987) emphasizes the situation model. With a plausibility strategy, encoding differences between explicit and implicit text ideas at other levels could be masked.

These considerations raise the question of the form that representational differences between explicit and implicit text ideas might take, and the origins of such differences. Contemporary theories and evidence offer at least three suggestions about these issues. First, Schmalhofer, McDaniel, and Keefe (2002) posited that because explicit ideas and not implicit ideas find direct expression in the text, only the former are encoded in the surface representation.

The situation model, in contrast, serves to integrate text information and general knowledge, so implicit as well as explicit ideas appear in it. However, it is likely that bridging inferences are more highly interconnected with other situational ideas than are elaborative inferences (Schmalhofer et al., 2002). That is, with PRESENT situationally computed upon the reading of Valerie left early for the birthday party, it is eligible to become interconnected with the ideas underlying the bridge-inducing sentence She spent an hour shopping at the mall. Second, a concomitant difference between explicit and implicit representations is that the latter may not be lexically specified at any level. In this regard, the fate of the actress who has fallen from the 14th story might be encoded as DEAD, DEPARTED, DECEASED, or even the indeterminate SOMETHING BAD HAPPENED (McKoon \& Ratcliff, 1986). Third, representational differences may also stem from the particular processes by which the reader derives the representations. Indeed, ideas, such as text inferences, that people generate from an original stimulus often have enhanced memorial status (Anderson \& Bower, 1972; Duffy, Shinjo, \& Myers, 1990; Slamecka \& Graf, 1978).

To summarize, several commonly used measures may inaccurately portray the LTM representations of explicit and implied text ideas as being equivalent. A potentially informative approach to this problem is the processdissociation procedure (Jacoby, 1991), an incisive technique for evaluating the memorial quality of an experience. Process dissociation rests on the dual-process assumption that memory retrieval is supported by a controlled contribution, called "recollection," and an automatic contribution, called "familiarity."

Contemporary theoretical analyses have been largely silent concerning the relative contributions of recollection and familiarity to text retrieval. This study was therefore designed to examine the process-dissociation profiles of explicit text ideas and text inferences in order to compare the quality of their LTM representations. The next section describes process dissociation and its application to these issues.

\section{Process Dissociation}

Paradigm. In a classic example of process dissociation, Jacoby's (1991, Experiment 3) participants encountered anagrams to solve and words to read in Phase 1 of an experiment. In Phase 2, they recited a distinct set of words that they heard. Phase 3 comprised a recognition test that presented Phase 1 and 2 words, and new words. Some participants received an inclusion instruction to label as "old" all words previously encountered in Phase 1 or 2.

Others received an exclusion instruction: They were directed to say "old" only to those words encountered in Phase 2. Jacoby proposed that, in inclusion, a Phase 1 word could be labeled "old" as a result of the independent impact of two processes: Controlled recollection of the word originating in Phase 1 (occurring with probability $c$ ) and automatic familiarity of the word exceeding a threshold for responding "old" (occurring with probability $a$ ).

Consequently, the probability of labeling a Phase 1 word "old" in inclusion, $O_{\mathrm{i}}$, is given by Formula 1. On the other hand, in exclusion, a Phase 1 word could be labeled "old" only if it was not recollected as originating in Phase 1 (by the exclusion instruction, recollection would result in a "new" response) but its familiarity exceeded the response threshold (Formula 2 ).

$$
\begin{array}{ll}
O_{\mathrm{i}}=c+a-c a=c+ & (1-c) a \\
O_{\mathrm{e}}= & (1-c) a
\end{array}
$$

Subtraction of Formula 2 from Formula 1 reveals that

$$
c=O_{\mathrm{i}}-O_{\mathrm{e}}
$$

Finally, substitution of this value for $c$ in Formula 2 yields

$$
a=O_{\mathrm{e}} /\left[1-\left(O_{\mathrm{i}}-O_{\mathrm{e}}\right)\right]
$$

Jacoby (1991, Experiment 3) reported that $c$ was considerably greater for anagrams than read words, but the difference between the $a$ values for the two was moderate.

Process-dissociation assumptions. The present study adopted the process-dissociation assumption that memory retrieval is supported by familiarity and recollection. The familiarity-recollection distinction is supported by several dissociations, as summarized in a large-scale review of the recognition memory literature (Yonelinas, 2002). Two dissociations are especially important for the present purposes. First, familiarity exhibits qualities of automatic processing, whereas recollection is controlled. In this regard, familiarity is associated with faster operating speeds (Hintzman \& Caulton, 1997; Reder, 1987) and lower demands on attentional resources (Dehn \& Engelkamp, 1997; Jacoby, Lindsay, \& Toth, 1992; Jacoby, Toth, \& Yonelinas, 1993; Komatsu, Graf, \& Uttl, 1995; Toth, 1996) than is recollection.

Second, familiarity is sensitive to both (1) perceptual manipulations of verbal material, such as those corresponding to manipulations of stimulus modality, font, and extent of maintenance rehearsal of words (Gardiner, Gawlik, \& Richardson-Klavehn, 1994; Levy et al., 1995; Toth, 1996); and (2) conceptual manipulations, such as 
those distinguishing full and divided attention (Jacoby et al., 1992; Jacoby et al., 1993) and deep versus shallow semantic processing (Dehn \& Engelkamp, 1997; Komatsu et al., 1995; Toth, 1996). Recollection, in contrast, is sensitive only to conceptual manipulations, and is more sensitive to such manipulations than is familiarity. Thus, it would seem that perceptual representations of verbal material support familiarity but not recollection; and conceptual (i.e., meaning-based) representations are supportive of both familiarity and recollection, but are more supportive of the latter than the former.

Yonelinas (2002) also highlighted that familiarity bears the qualities of a continuous measure of memory strength (and thus a signal-detection mechanism), whereas recollection depends on detecting associations between the test probe and the learning context ( $a$ threshold mechanism; Yonelinas, 2001, 2002). He also reviewed evidence that familiarity and recollection exhibit different electrophysiological profiles and different responses to brain injury. Finally, it is noteworthy that other experimental paradigms provide converging evidence for the dualprocess assumption. For example, participants' judgments that they either remember or just "know" that a retrieval probe occurred earlier are proposed to, respectively, reflect recollection and familiarity (Gardiner \& Java, 1991; Tulving, 1985).

The extended process-dissociation procedure. Some have argued that Jacoby's (1991) analysis has the limitations of (1) resting on the assumption that controlled and automatic processes operate independently and (2) not taking guessing processes into account (Graf \& Komatsu, 1994; Joordens \& Merikle, 1993). For these reasons, we used the extended process-dissociation analysis (Buchner, Erdfelder, \& Vaterrodt-Plunnecke, 1995). Buchner et al. redefined the automatic parameter $a$ as the conditional probability that the familiarity of a Phase 1 word exceeds the response threshold given that the word is not recollected as originating in Phase 1. Determining the value of this conditional probability does not require the assumption of the independence of the controlled and automatic processes. ${ }^{1}$ In addition, Buchner et al. introduced the guessing parameters $g_{\mathrm{i}}$ and $g_{\mathrm{e}}$ for inclusion and exclusion, respectively.

Buchner et al. (1995) presented their analysis in the form of a multinomial processing tree model, identifying all of the processing routes that can result in "old" and "new" responses in any given experimental condition. For example, Buchner et al. assumed that, in inclusion, a Phase 1 word would be labeled "old" if it was recollected as originating in Phase $1(c)$; it was not recollected but its familiarity exceeded the response threshold $[(1-c) a]$; or, failing that, it was guessed to be an old word $[(1-c)$ $\left.(1-a) g_{i}\right]$. Consequently, the probability of labeling a Phase 1 word "old" in inclusion is given by Formula 5. A comparable formula (6) is derived for $O_{\mathrm{e}}$.

$$
\begin{aligned}
& O_{\mathrm{i}}=c+(1-c) a+(1-c)(1-a) g_{\mathrm{i}} \\
& O_{\mathrm{e}}=(1-c) a+(1-c)(1-a) g_{\mathrm{e}} .
\end{aligned}
$$

Finally, Buchner et al. (1995) assumed that new words are never recollected as originating in Phase 1, and their familiarity never exceeds the response threshold. Consequently, the guessing parameters $g_{\text {, }}$ and $g_{\mathrm{e}}$ are simply equal to the probabilities of responding "old" to new words in inclusion and exclusion, respectively. As a result, Equations 5 and 6 can be solved for the parameters $c$ and $a$.

Process dissociation and text retrieval. We propose that process dissociation is as relevant to text retrieval as to the retrieval of other material. First, to assume otherwise would entail creating task-specific retrieval theories devoid of psychological generality (Singer \& Kintsch, 2001). Second, there is considerable evidence that text retrieval is supported by both recollection and familiarity. In question answering and text recognition at short testing delays, readers can confidently identify test probes with the text, in a manner consistent with conscious recollection (Reder, 1982). In a study of Hasher and Griffin (1978), some participants were informed that a text they had read a week earlier had been accompanied by the incorrect title. The participants recalled six times as many explicit text ideas in the title-incorrect condition than in a control title-correct condition. This striking result suggests that, unable to reconstruct text ideas on the basis of its title (e.g., "Going Hunting"), the title-change participants scrutinized their text representations in a manner that resulted in the recollection of some ideas from the text.

Familiarity likewise appears to contribute to text retrieval. For example, reading time is lower for a story that repeats, in new words, the theme of a prior text than for unrelated stories and for stories that repeat words but present a novel theme (Levy et al., 1995; Masson, 1993). Ordinary reading is not an explicit memory task, so the enhancement of comprehension fluency is more likely attributable to the familiarity of the text ideas than to an experience of recollection. Second, the "feeling of knowing," or familiarity, of a queried text idea affects the strategy and duration of memory search (Reder, 1987). Consistent with these observations, Long and Prat (2002) reported that readers' background knowledge affected the recollective but not the familiarity contribution to text recognition. This outcome clarified prior findings that knowledge affects text recall but not recognition (e.g., Moravcsik \& Kintsch, 1993).

Process dissociation and text inferences. Recognition and other positive evaluations about probes that represent text inferences represent a normal state of affairs rather than an anomaly. Indeed, the acceptance of related distractors (Brainerd, Reyna, \& Mojardin, 1999) and prototypes (Clark \& Gronlund, 1996) is a central phenomenon of human memory. In this regard, people make positive recognition judgments about (1) numerous classes of nonpresented associates of words encountered in lists (Underwood, 1965), (2) items that best capture the convergence among the meanings of listed words (Deese, 1959; Roediger \& McDermott, 1995), and (3) test sentences that express the interrelation among other sentences (Bransford \& Franks, 1971). Text-inference recognition 
probes, by definition, do not appear in their antecedent texts. Therefore, the notion of a recollective contribution to inference retrieval merits scrutiny.

The widely considered construct of misrecollection is pertinent to this issue. Specifically, initial study may be accompanied either by the retrieval of a cohort of items associated with a list item (Underwood, 1965) or by the representation of a prototype of a set of related list items (e.g., a sentence that integrates the meaning of several other sentences; Clark \& Gronlund, 1996). Later, at retrieval, the presentation of an associate or prototype of the list items may access these representations and result in misrecollection. Yonelinas $(2002$, p. 465$)$ noted that "subjects may recollect that the item was encountered but fail to recollect that it was self-generated rather than studied." Yonelinas (2002) proposed that even without the initial representation of the prototype, adequate similarity between the probe and multiple list items is sufficient to yield misrecollection.

\section{Overview}

We applied the extended process-dissociation procedure (Buchner et al., 1995) to the study of text inferences. In Phase 1 of each experiment, participants read sentences or short texts, some containing explicitly stated ideas and others affording the opportunity to draw specific inferences. In Phase 2, participants read words aloud. In Phase 3, under inclusion or exclusion instructions, participants performed recognition judgments about Phase 1 target words, Phase 2 words, and new words. Recognition judgments in Phase 3 permitted the estimation of the contributions of controlled and automatic processes to the recognition of words representing explicit and implicit text ideas.

Several features of the results were of particular interest. Most generally, if there are systematic differences in the representation of explicit and implicit text ideas, then their process-dissociation profiles would be expected to differ. Such differences, coupled with the interference from the Phase 2 list and the delayed nature of Phase 3 recognition, would diagnose dissimilarities between the LTM representations of explicit and implicit text ideas.

The controlled and automatic components of process dissociation each merited close scrutiny. As discussed earlier, recollection (the controlled influence) appears to be exclusively supported by conceptual representations. Therefore, detecting a greater controlled contribution to the recognition of explicit as opposed to implicit text ideas would tend to indicate a richer conceptual representation of the explicit ideas.

Evaluating the automatic, or familiarity-based, component of process dissociation is more complex. Familiarity is proposed to reflect both conceptual and perceptual influences. However, perceptual processes (e.g., word identification; Perfetti, 1989) cannot be relevant to text inferences, the corresponding words of which are absent from the text. Therefore, a greater automatic contribution to explicit than to implicit retrieval might result from the absence of perceptual representations of implied concepts. However, such an interpretation is complicated by the possibility that the distinct qualities of the conceptual representation of implicit text ideas (Duffy et al., 1990) might offset their meagre perceptual representation.

There is also the possibility that by Phase 3, the perceptual representation of explicit ideas has degraded and may no longer be supportive of familiarity. These issues will receive further consideration later.

Finally, we predicted that the false alarm rate, and corresponding guessing parameter, would be greater in inclusion than in exclusion. By virtue of the processdissociation instructions, there is an appreciably higher ratio of "old" to "new" responses in inclusion than in exclusion. This promotes a stronger bias for guessing "old" in inclusion than in exclusion (Buchner et al., 1995; Dehn \& Engelkamp, 1997; Graf \& Komatsu, 1994; cf. Toth, Reingold, \& Jacoby, 1994). This comparison provided a point of contact with prior investigations.

\section{EXPERIMENT 1}

Experiment 1 examined the process-dissociation profiles of explicit text ideas and corresponding elaborative inferences. In particular, we scrutinized sentences that stated or implied a case-filling element (Fillmore, 1968). For example, the agent mailman is respectively explicit and implicit in The mailman delivered the letter in the rain and The letter was delivered in the rain. These sentences were presented in Phase 1 of the process-dissociation procedure, and the target mailman later appeared in Phase 3. The Phase 3 recognition responses permitted the estimation of the controlled and automatic contributions to the recognition of explicit and implicit targets.

\section{Method}

\section{Participants}

The participants were 154 female and male native English speaking students of introductory psychology. They took part in partial fulfillment of a course requirement.

\section{Materials}

Phase 1. The materials were four counterbalanced lists derived from 48 experimental sentences. Twenty-four sentences included a high-probability case-filling target word (e.g., mailman in 2a), and the other 24 included a low-probability target word (e.g., poet in $3 \mathrm{a}$ ).

(2a) The mailman delivered the letter in the rain.

(3a) The poet broke the television with the brick.

The target words played the role of agent, object, or instrument in their sentences. The high-probability elements were identified as the first choice concept to fill a specified role in a fact (e.g., the agent for deliver the letter) by a mean of $88.6 \%$ of participants (Singer, 1980). The low-probability elements, in contrast, were identified by Singer (1981) as possible but not highly likely case-filling elements on the basis either of Singer's (1980) norms or intuition.

Experimental sentences could appear in the explicit condition (e.g., 2a, 3a) or in the implicit condition, with the target word removed (e.g., 2b, 3b):

(2b) The letter was delivered in the rain.

(3b) The television was broken with the brick. 
Finally, in a third condition, called "absent," a given sentence did not appear in Phase 1. The target words from the absent sentences functioned as distractor words in Phase 3.

The low-probability condition particularly contrasted with the high-probability condition in that there is no reason for poet to be part of the LTM representation of sentence (3b). Therefore, we predicted that both the controlled and automatic contributions to recognition would approximate zero in the low-probability implicit condition. Although this outcome would likely be no different from recognition in the absent condition, the low-probability implicit condition was included in order to test these hypotheses and for the sake of the completeness of the design.

In the first of four counterbalanced lists, high-probability sentences were randomly assigned to conditions in the frequencies 6 explicit, 6 implicit, and 12 absent. Parallel assignments were made for the low-probability sentences. Then, each sentence was assigned to a random position in the list, subject to the restrictions that half of the sentences in each condition appear in each half of the list and that no more than three sentences from the same condition appear consecutively. Absent sentences held "virtual" positions in the lists and explicit and implicit sentences appeared around them. List 1 thus included only 24 experimental sentences (the other 24 were absent). List 1 began with 4 buffer sentences and ended with 2 more. The buffer sentences, half explicit and half implicit in their form, were drawn at random from the same pool as the experimental sentences.

Lists 2 to 4 were constructed by cycling the sentences across conditions, following a Latin-square design. This resulted in each sentence appearing in the explicit and implicit conditions in one list each, and in the absent condition in two lists.

Phase 2. The Phase 2 words were 36 nouns drawn at random from sentences from the same pool as the Phase 1 experimental sentences. None of the 36 nouns appeared in Phase 1 sentences. The Phase 2 words were preceded by 4 buffer words.

Phase 3. The Phase 3 recognition list consisted of the 24 target words of the explicit and implicit sentences of Phase 1; the 36 words of Phase 2; and 24 distractor words: namely, the target words from those sentences that had been assigned to the absent condition in Phase 1. These 84 words were preceded by 6 buffer words: 2 from the buffer sentences of Phase 1, 2 Phase 2 buffer words, and 2 new words. The Phase 3 words were arranged in a single random order.

\section{Procedure}

The participants were tested in groups of 1 to 4 in separate, closed rooms. Each sat at a computer station consisting of a personal computer, monitor, and keyboard. They were randomly assigned in approximately equal numbers to the eight conditions obtained by crossing list ( 1 to 4 ) and instruction (inclusion or exclusion). There were three phases of testing. Whether the participant was in the in clusion or exclusion condition pertained only to Phase 3 . The participant was informed at the outset that the experiment would involve several tasks.

Phase 1. Phase 1 involved the presentation of the experimental sentences. On each trial, the word READY appeared in the center of row 4 of the screen and was removed when participants pressed the space bar to begin. A fixation point was then presented for $500 \mathrm{msec}$ in column 1 of row 5 , followed immediately by the sentence. The participants rated the degree of activity conveyed by the sentence on a 4 -point scale $(1=$ very passive, $4=$ very active $)$. They used the four fingers of their left hand to press the corresponding numeric keys at the top left of the keyboard. Following a response, the sentence was removed, and the next trial began $3 \mathrm{sec}$ later.

Phase 2. The participant pressed a key to initiate Phase 2, and a fixation point then appeared for $500 \mathrm{msec}$ at column 1 of row 5 . Then, the Phase 2 words were displayed for $2,000 \mathrm{msec}$ each, plus a 50-msec interword interval. The participant read each word aloud. These responses were registered by a tape recorder.
Phase 3. Phase 3 constituted a recognition task. In the inclusion condition, the participants were instructed to label a test word "old" if it had appeared either in a Phase 1 sentence or in Phase 2, and "new" otherwise. In exclusion, the participants were instructed to label a test word "old" only if it had appeared in Phase 2. The exclusion instructions specified that if the participant remembered that a test word had appeared in Phase 1, it was to be labeled "new," because no word had occurred in both Phases 1 and 2. The participant pressed a key to begin Phase 3. On each trial, a fixation point was presented for $500 \mathrm{msec}$, followed by the test word. The participant labeled the word either "new" or "old," using keys " $x$ " (left index finger) and "." (right index finger), respectively. When the response was registered, the word disappeared. After a 1 -sec intertrial interval, the next trial began. If no response was made within $10 \mathrm{sec}$, the trial terminated, and a "no" response was credited.

\section{Results}

\section{Target Recognition Rates}

The rates of responding "old" to Phase 1 target words in the Phase 3 recognition test appear in Table 1. Analysis of variance (ANOVA) was applied to these values, alternately treating participants $\left(F_{1}\right)$ and items $\left(F_{2}\right)$ as the random effect. In the participants-random ANOVA, relation (explicit, implicit, absent) and probability (high, low) were within-participants variables and instruction (inclusion, exclusion) was a between-participants variable. In the items-random ANOVA, relation and instruction were within-items variables and probability was a betweenitems variable. A significance level of $\alpha=.05$ was used unless otherwise indicated.

Because our main focus was on the multinomial model analyses, we present the statistics only for the significant recognition-rate ANOVA effects. Those statistics appear in Appendix A.

Recognition rates varied significantly with relation, and were significantly higher under inclusion than under exclusion instructions. The relation $x$ instruction interaction was significant, diagnosing a greater relation effect in inclusion than in exclusion. Both the relation $X$ probability and instruction $X$ probability interactions were also significant. These effects appear to result from the relatively high recognition rate in the implicit highprobability condition. The relation $\times$ instruction $\times$ probability interaction was not significant.

A test of simple main effects revealed an effect of instruction in the absent condition $\left[F_{1}(1,152)=24.80\right.$, $\left.M S_{\mathrm{e}}=0.04 ; F_{2}(1,46)=51.64, M S_{\mathrm{e}}=56\right]$. This indicated that the false alarm rate was higher in inclusion than in exclusion.

Table 1

Mean Recognition Rates of Phase 1 Target Words in Phase 3 of Experiment 1

\begin{tabular}{lcccccc}
\hline & \multicolumn{3}{c}{ High Probability } & & \multicolumn{3}{c}{ Low Probability } \\
\cline { 2 - 3 } \cline { 5 - 7 } Instruction & Expl & Impl & Abs & Expl & Impl & Abs \\
\hline Inclusion & .57 & .34 & .23 & .54 & .19 & .19 \\
Exclusion & .15 & .10 & .10 & .18 & .06 & .09 \\
\hline
\end{tabular}

Note-The relations were explicit (Expl), implicit (Impl), or absent (Abs). 


\section{Model Analyses}

The processing tree model of Experiment 1 appears in Appendix B. Parameter estimation and hypothesis testing were performed using the general processing tree program of $\mathrm{Hu}$ and Phillips (1999). The frequencies of "old" and "new" responses under each experimental condition, pooled across participants, appear in Appendix $\mathrm{C}$. The model and the frequencies were entered into the program. The evaluation of a null hypothesis (e.g., $c_{\mathrm{EH}}=c_{\mathrm{IH}}$ or $c_{\mathrm{IH}}=0$ ) was performed by subtracting the chi-square goodness-of-fit statistic, $G^{2}$, for the full model in Appendix B, from $G^{2}$ for the submodel constrained by the null hypothesis. ${ }^{2}$ The difference between these values is also a $G^{2}$ statistic. It has one degree of freedom because the full model and submodel differ by one free parameter. If the difference exceeds the chi-square critical value of 3.84 , then the constraint imposed by the null hypothesis significantly reduces the fit of the full model and so the null hypothesis is rejected (for the details about hypothesis testing using processing tree models, see Batchelder \& Riefer, 1990; Hu \& Batchelder, 1994; Hu \& Phillips, 1999). The $G^{2}$ difference statistic is the statistic that is reported below.

The resulting parameters are shown in Table 2 . We evaluated a limited number of hypotheses that compared the parameters to one another and to 0 . For high-probability targets, $c$ was greater in the explicit than in the implicit condition $\left(G^{2}=23.64\right)$, which in turn was greater than $0\left(G^{2}=14.69\right)$. Parameter $a$ was also greater in the explicit than the implicit condition $\left(G^{2}=20.54\right)$, but the latter did not differ significantly from $0\left(G^{2}=0.90\right)$. Thus the process-dissociation profiles for explicit and implicit targets differed.

For low-probability targets, $c$ was greater in the explicit than the implicit condition $\left(G^{2}=51.75\right)$, and the latter had the numerical value $0\left(G^{2}=0.01\right)$. Likewise, $a$ was greater in the explicit condition than in the implicit condition $\left(G^{2}=69.19\right)$, and the latter had the numerical value $0\left(G^{2}=0.02\right)$. As expected, the contributions of controlled and automatic processes to the recognition of targets in the low-probability implicit condition were zero.

Comparisons between the high- and low-probability conditions revealed that $c$ was significantly greater in the high-probability than in the low-probability condition

Table 2

Parameter Estimates in Experiment 1

\begin{tabular}{|c|c|c|c|c|}
\hline \multirow[b]{2}{*}{ Parameter } & \multicolumn{2}{|c|}{ High Probability } & \multicolumn{2}{|c|}{ Low Probability } \\
\hline & Explicit & Implicit & Explicit & Implicit \\
\hline$c$ & $.34^{*}$ & $.13^{+}$ & $.30^{*}$ & .00 \\
\hline$a$ & $.15^{*}$ & .02 & $.19^{*}$ & .00 \\
\hline
\end{tabular}

Note-The superscripts denote statistical comparisons among levels of the relation variable (explicit, implicit, absent) only. All alpha levels for those comparisons were .05 . The guessing parameters $g_{i}$ and $g_{e}$ were, respectively, .23 and .10 in the high-probability condrtion and .19 and .08 in the low-probability condition. "Explicit statistically higher than implicit. IImplicit statistically higher than 0 . for implicit targets $\left(G^{2}=8.67\right)$ but not for explicit targets $\left(G^{2}=0.94\right)$. The parameter $a$ did not differ significantly between the high- and low-probability conditions for either explicit targets $\left(G^{2}=1.87\right)$ or implicit targets $\left(G^{2}=0.91\right)$. Thus, the process-dissociation profiles were similar for high- and low-probability targets that were explicit.

\section{Discussion}

In the high-probability condition, the recognition of explicit targets reflected both controlled and automatic influences, whereas implicit-target recognition reflected a smaller controlled influence and no automatic influence. These results supported our proposal of differences between the LTM representation of explicit text information and elaborative inferences. Moreover, the difference between the controlled influences for explicit ideas and elaborative inferences suggests a more robust conceptual representation in the former condition. This is because the controlled contribution is supported exclusively by conceptual processes. This difference could mean that extra support for explicit recollection is provided either by the lexical components of the propositional textbase or by the text situation model. These alternatives will be considered in the General Discussion section.

The absence of an automatic influence in the implicit condition tends to deny the presence of perceptual representations of the implicit concepts. This is because the automatic influence is supported in part by perceptual representations (Yonelinas, 2002). ${ }^{3}$ This outcome is consistent with the readers' lack of opportunity to perceptually process the implied concepts.

In the explicit condition, in contrast, a significant automatic contribution was measured. Because the automatic contribution reflects both perceptual and conceptual representations and the explicit concepts presumably received perceptual processing, it is tempting to conclude that the automatic component was partially supported by perceptual representations. However, we cannot rule out the possibility that by Phase 3, the perceptual representation of explicit ideas had degraded and no longer supported familiarity, and the explicit automatic contribution exclusively reflected robust conceptual representations. This issue will receive further scrutiny in the discussion of Experiment 3.

It is noteworthy that the significant controlled contribution to implicit recognition could result either from encoding of those inferences during the reading of the original text or from inferentially relating the recognition probes to the antecedent text representations at retrieval time (Corbett \& Dosher, 1978; McKoon \& Ratcliff, 1986). Although people routinely incorrectly recognize elaborative-inference probes (Johnson, Bransford, \& Solomon, 1973; Singer, 1980), the evidence indicates that the corresponding inferences are not reliably encoded during reading (McKoon \& Ratcliff, 1986; Potts et al., 1988; Singer \& Ferreira, 1983). Experiment 1 further analyzed the retrieval processes that result in the recognition of 
elaborative-inference probes: The results indicated that inference recognition was exclusively supported by controlled influences. However, Experiment 1 does not particularly bear on the temporal locus of the computation of text inferences.

Two other features of the results reconcile Experiment 1 with logical analysis and prior findings. First, both $c$ and $a$ were zero in the low-probability implicit condition. This is consistent with the absence of any basis for recognizing poet in the context of The television was broken with the brick. Second, as predicted, the false alarm rate, and corresponding guessing parameter, was higher in inclusion than exclusion.

\section{EXPERIMENT 2}

Having presented evidence that explicit text ideas and elaborative inferences have different LTM representations, we next focused on bridging inferences. Experiments 2 and 3 examined bridging inferences of characters' goals and motives (e.g., Dopkins, Klin, \& Myers, 1993; Klin, 1995; Myers et al., 1987; Singer \& Halldorson, 1996). For example, upon reading Valerie left early for the birthday party. She spent an hour shopping at the mall, people infer that the second action serves a goal of the first-namely, the purchase of a present.

\section{Method}

\section{Participants}

The participants were 136 naive individuals from the same pool that was used in Experiment 1. Sixty-eight participants were randomly assigned to each of the inclusion and exclusion conditions.

\section{Materials}

Overview. The Phase 1 materials were derived from 16 twosentence texts, each explicitly stating a highly probable target concept in the second sentence (e.g., present in $4 \mathrm{a}$, below). The experimental texts could appear in one of four conditions-explicit, motive inference, control, and absent. The text ( $4 \mathrm{~b})$, the motive inference sequence, implied but did not explicitly state that Valerie shopped for a present. The control text (4c) was highly similar to (4b), but suggested the crucial inference less or not at all. Finally, a text could be entirely absent in Phase 1 .

(4a) Valerie left early for the birthday party. She spent an hour shopping for a present at the mall. (explicit)

(4b) Valerie left early for the birthday party. She spent an hour shopping at the mall. (motive inference)

(4c) Valerie left the birthday party early. She spent an hour shopping at the mall. (control)

The large volume of text that had to appear in Phase 1 prompted us to conduct this experiment in four process-dissociation blocks. In Phase 1 of each block, the participant evaluated the degree of activity conveyed by each of five two-sentence texts. In Phase 2, 11 words were read aloud from the monitor screen. In Phase 3, the participant had to recognize the Phase 1 target words, Phase 2 words, and distractor words. Here are the details:

Phase 1. The Phase 1 materials were four counterbalanced lists. Each list comprised four blocks of trials. List 1 was constructed as follows: First, the 16 experimental texts were randomly assigned in equal numbers to the explicit, motive, control, and absent conditions.

Second, the texts were randomly assigned to the blocks, subject to the restriction that there be one text from each condition in each block. Each text was also randomly assigned to a fixed position in its block. Texts in the absent condition simply did not appear in the Phase 1 list.

Third, each block began and ended with a buffer text of the same form as the experimental texts, chosen at random from a list of 28 filler passages used by Singer and Halldorson (1996, Experiment 1). Lists 2 to 4 were constructed by cycling the List 1 experimental texts across conditions, using a Latin-square procedure.

Phase 2. In each block, the Phase 2 list consisted of nine words, preceded and followed by one buffer word. All of these words were selected at random from Singer and Halldorson's (1996, Experiment 1) filler passages. Across the four blocks, the nonbuffer words comprised 24 nouns, 6 verbs, and 6 adjectives, yielding proportions approximately equal to those of the target words of Phase 1 . No Phase 2 word appeared in any Phase 1 text.

Phase 3. The Phase 3 recognition list for each block began with the presentation of 3 practice words. Two of the practice words had appeared in the Phase 1 buffer texts, and the other one was new. The remainder of the list consisted of the 4 target words ( 1 per condition), 4 of the Phase 2 words, and 2 new words. The new words were selected from Singer and Halldorson's (1996, Experiment 1) filler passages and appeared nowhere else in the experiment. The 10 nonpractice words were presented in a fixed random order.

\section{Procedure}

In Phase 1 of each of the four blocks, each trial began with the appearance of the word READY in the middle of row 4 of the screen. The participant pressed the keyboard space bar to begin the trial. A fixation point was then displayed for $500 \mathrm{msec}$ at column 1 , row 5 of the screen, followed by the first sentence of the text. The participant pressed the space bar again to signal comprehension of the first sentence, which resulted in the removal of that sentence. After a $50-\mathrm{msec}$ interstimulus interval, the second sentence appeared. The participant then rated the activity conveyed by the text, in the same manner as in Experiment 1. The trial chronologies in Phases 2 and 3 were identical to those of Experiment 1.

\section{Results}

\section{Target Recognition Rates}

The rates of responding "old" to Phase 1 target words in the Phase 3 recognition test appear in Table 3. In a participants-random ANOVA, relation (explicit, motive, control, absent) was a within-participants variable and instruction (inclusion, exclusion) was a betweenparticipants variable. In an items-random ANOVA, both relation and instruction were within-items variables. There were significant main effects of instruction $\left[F_{1}(1,134)=\right.$ 69.72, $\left.M S_{\mathrm{e}}=0.11 ; F_{2}(1,15)=53.6, M S_{\mathrm{e}}=259.1\right]$ and relation $\left[F_{1}(3,402)=42.07, M S_{\mathrm{e}}=0.03 ; F_{2}(3,45)=23.6\right.$, $\left.M S_{\mathrm{e}}=5,100.3\right]$. The instruction $\times$ relation interaction was also significant $\left[F_{1}(3,402)=23.45, M S_{\mathrm{e}}=0.03\right.$;

Table 3

Mean Recognition Rates of Phase 1 Target Words in Phase 3 in Experiments 2 and 3

\begin{tabular}{clcccc}
\hline & & \multicolumn{4}{c}{ Relation } \\
\cline { 3 - 6 } Experiment & Instruction & Explicit & $\begin{array}{c}\text { Motive } \\
\text { Inference }\end{array}$ & Control & Absent \\
\hline 2 & Inclusion & .57 & .34 & .24 & .16 \\
3 & Exclusion & .13 & .06 & .10 & .05 \\
3 & Inclusion & .71 & .39 & .29 & .18 \\
& Exclusion & .13 & .09 & .11 & .06 \\
\hline
\end{tabular}


$\left.F_{2}(3,45)=16.7, M S_{e}=1,793.9\right]$. The interaction indicates that the relation effect was greater in the inclusion condition than in the exclusion condition. The inclusion condition bore some resemblance to prior inspections of bridging-inference recognition. Therefore, contrasts were performed for the levels of the relation variable in that condition. The inclusion recognition rate was higher in the explicit than in the motive condition $\left[F_{1}(1,63)=\right.$ $\left.31.56, M S_{\mathrm{e}}=1.00 ; F_{2}(1,15)=18.09, M S_{\mathrm{e}}=253.43\right]$, which in turn was higher than in the control condition $\left[F_{1}(1,63)=6.58, M S_{\mathrm{e}}=.65 ; F_{2}(1,15)=12.47, M S_{\mathrm{e}}=\right.$ 47.73], which in turn was higher than in the absent condition $\left[F_{1}(1,63)=7.81, M S_{\mathrm{e}}=0.50 ; F_{2}(1,15)=6.47\right.$, $\left.M S_{\mathrm{e}}=105.83\right]$.

In the absent condition, mean acceptance rates of .16 and .05 were measured in the inclusion and exclusion conditions, respectively, values that differed significantly $\left[F_{1}(1,134)=12.38, M S_{\mathrm{e}}=.03 ; F_{2}(1,15)=20.05, M S_{\mathrm{e}}=\right.$ 39.79].

\section{Model Analyses}

The processing tree model is presented in Appendix D, and the frequencies of old and new responses, pooled across participants and the four process-dissociation blocks, are shown in Appendix C. The modeling analyses were performed as in Experiment 1. The resulting parameter estimates are displayed in Table 4. Parameter $c$ of the explicit condition exceeded that of the motive condition $\left(G^{2}=12.50\right)$, which in turn exceeded that of the control condition $\left(G^{2}=8.97\right)$, which did not significantly exceed zero $\left(G^{2}=1.19\right)$. Parameter $a$ of the explicit condition exceeded those of both the motive condition $\left(G^{2}=16.62\right)$ and the control condition $\left(G^{2}=\right.$ 9.60). The latter two $a$ values did not differ significantly $\left(G^{2}=1.63\right)$. However, $a$ was significantly greater than zero in the control condition $\left(G^{2}=6.04\right)$, but not in the motive condition $\left(G^{2}=1.08\right)$.

\section{Discussion}

Different process-dissociation profiles were detected in the explicit and the implicit conditions. This tends to

Table 4

Parameter Estimates in Experiments 2 and 3

\begin{tabular}{ccccc}
\hline & & \multicolumn{3}{c}{ Relation } \\
\cline { 3 - 4 } Experiment & Parameter & Explicit & $\begin{array}{c}\text { Motive } \\
\text { Inference }\end{array}$ & Control \\
\hline 2 & $c$ & $.39^{*}$ & $.20^{\dagger}$ & .05 \\
& $a$ & $.16^{*}$ & .02 & $.06 \ddagger$ \\
3 & $c$ & $.55^{*}$ & $.21^{\dagger}$ & $.09 \$$ \\
& $a$ & $.24^{*}$ & $.06 \pm$ & $.06 \ddagger$ \\
\hline
\end{tabular}

Note--The alpha level for the statistical comparisons in this table was .05 unless otherwise indicated. The guessing parameters $g_{\mathrm{i}}$ and $g_{\mathrm{e}}$ were, respectively, .16 and .05 in Experiment 2 and .18 and .06 in Experiment 3. "Explicit statistically higher than motive. tMotive statistically higher than control. ‡Value statistically higher than 0 . §Value marginally higher than zero, $.05<p<.10$. suggest differences between the LTM representation of explicit text information and bridging inferences.

Consider the controlled contribution (c) to recognition. First, explicit recognition received greater controlled support than did implicit recognition. This suggests that explicitly stated concepts received stronger conceptual encoding than did motive-bridging inferences, because $c$ is proposed to reflect only conceptual representations (Yonelinas, 2002). The basis of this conceptual difference will be addressed in the General Discussion. Second, $c$ was, in turn, greater in the motive than in the control condition. This outcome is consistent with our basic contention that motive inferences have a special status in text representation (McDaniel, Schmalhofer, \& Keefe, 2001; Potts et al., 1988; Singer \& Halldorson, 1996; Singer et al., 1992).

Parameter $a$ of the explicit condition exceeded that of the implicit condition, which did not significantly exceed zero. As in Experiment 1, this pattern suggests that implicit recognition received no support from perceptual representations, but it cannot diagnose the relative contribution to explicit recognition of perceptual and conceptual representations.

Parameter $a$ did not differ between the motive and control conditions, but $a$ was significantly greater than zero in the control condition, whereas it was not in the motive condition. We judged this result to be equivocal and delay its examination until Experiment 3.

\section{EXPERIMENT 3}

We considered Experiment 2 to merit replication. Experiment 3 served that purpose, but it also implemented a priming procedure. In particular, in the Phase 3 recognition lists, the target words were immediately preceded by words that had appeared explicitly in their respective texts. The prime is considered to augment the tendency for the target word to cue the relevant text representation (McKoon \& Ratcliff, 1988; Singer \& Halldorson, 1996). Failure to access that representation could distort the process-dissociation patterns under inspection. Using the unprimed procedure of Experiment 2, our measurements might represent the averaging of trials on which the targets accessed their texts and other trials on which they did not. For example, the incorrect interpretation of an ambiguous probe such as present might impede the retrieval of the antecedent text. This, in turn, could result in misleading estimates of recollection and familiarity in our experimental conditions.

\section{Method}

The participants were 127 individuals from the same pool that was sampled for the previous experiments. Sixty-three and 64 participants were randomly assigned to the inclusion and exclusion conditions, respectively. As in Experiment 2, the materials were arranged in four blocks. In each phase of each block, the materials were identical to those of Experiment 2 with the following exception: In Phase 3, each target word was preceded by a priming word. The priming words were nouns that stemmed from the first sen- 
tence of their texts. For example, for the motive inference text Sharon was eager to get the sports car. She went straight to the bank (target-loan), the priming word was car.

The inclusion of the primes increased the number of words in the Phase 3 lists of each block to 14, preceded by 3 practice words. Prime words from the texts in the absent condition were included in Phase 3. The procedure was identical to that of Experiment 2.

\section{Results}

\section{Target Recognition Rates}

The mean recognition rates are shown in Table 3 . ANOVA, using the same design as in Experiment 2, revealed main effects of instruction $\left[F_{1}(1,125)=101.80\right.$, $\left.M S_{\mathrm{e}}=0.11 ; F_{2}(1,15)=59.4, M S_{\mathrm{e}}=11.7\right]$ and of relation $\left[F_{1}(3,375)=59.14, M S_{\mathrm{e}}=0.03 ; F_{2}(3,45)=41.6\right.$, $\left.M S_{\mathrm{e}}=3.8\right]$. The instruction $\times$ relation interaction was significant $\left[F_{1}(3,375)=38.75, M S_{\mathrm{e}}=.03 ; F_{2}(3,45)=\right.$ $23.4, M S_{\mathrm{e}}=3.8$ ]. Contrasts ordered the inclusion acceptance rates higher in the explicit condition than in the motive condition $\left[F_{1}(1,59)=68.88, M S_{\mathrm{e}}=.77\right.$; $\left.F_{2}(1,15)=32.03, M S_{\mathrm{e}}=831.18\right]$, which in turn exceeded the control condition $\left[F_{1}(1,59)=7.45, M S_{\mathrm{e}}=\right.$ $\left..64 ; F_{2}(1,15)=7.67, M S_{\mathrm{e}}=191.73\right]$, which in turn exceeded the absent condition $\left[F_{1}(1,59)=10.64, M S_{\mathrm{e}}=\right.$ $\left..64 ; F_{2}(1,15)=6.88, M S_{\mathrm{e}}=428.01\right]$. Finally, there was a significant effect of instruction in the absent condition $\left[F_{1}(1,125)=11.13, M S_{\mathrm{e}}=0.04 ; F_{2}(1,15)=12.6, M S_{\mathrm{e}}=\right.$ 2.4]. The latter outcome again confirmed higher false alarm rates in inclusion than in exclusion.

\section{Model Analyses}

The processing tree model appears in Appendix D and the pooled frequencies of old and new responses are shown in Appendix C. Table 4 displays the resulting parameters. Parameter $c$ in the explicit condition exceeded that in the motive condition $\left(G^{2}=33.42\right)$, which in turn exceeded that of the control condition $\left(G^{2}=5.16\right)$, which in turn was marginally greater than zero $\left(G^{2}=3.38, p=\right.$ $.08)$. Explicit parameter $a$ exceeded both that of the motive condition $\left(G^{2}=15.28\right)$ and of the control condition $\left(G^{2}=17.20\right)$, and the latter two did not differ $\left(G^{2}=.01\right)$. However, $a$ differed significantly from zero both in the motive and control conditions (motive, $G^{2}=5.15$; control, $G^{2}=5.02$ ).

To assess the impact of the prime on the parameters, the processing tree models of Experiments 2 and 3 were combined and the corresponding parameter values compared. Parameter $c$ in the explicit condition was greater in Experiment 3 than in Experiment $2\left(G^{2}=7.36\right)$. There were no other significant differences.

\section{Discussion}

The qualitative pattern of the results resembled that of Experiment 2 . As before, the explicit $>$ motive $>$ control ordering of the controlled contribution $(c)$ indicates that the conceptual representation of the motive-bridging inference was, in some manner, weaker than for explicit ideas but privileged compared with the control condition concepts.
The joint analysis of Experiments 2 and 3 bears on the comparison between the conceptual representations for the explicit versus implicit conditions. The joint analysis indicated that the priming manipulation was associated with an increase in the controlled contribution $(c)$ to explicit recognition and no other parameter. This implicates conceptual representations as the locus of this increase, because $c$ is proposed to reflect only conceptual processes. Thus, it signals the presence of differences between the conceptual representation of explicit ideas and the motive-bridging inferences-otherwise, $c$ ought to have increased as much in the motive-inference condition as in the explicit condition. With regard to the motive versus control comparison, the fact that the priming procedure did not benefit the motive condition relative to the control condition suggests that the motive condition was not particularly disadvantaged by the unprimed procedure of Experiment 2. The possibility of such a disadvantage was raised in the introduction to Experiment 3.

The small familiarity component in the motive condition, like its null counterparts of Experiments 1 and 2, implies sparse perceptual representations of these bridging inferences. In contrast, the appreciable familiarity component of the explicit conditions could have been the result of either conceptual or perceptual representations. Of these alternatives, the effects of priming suggest that familiarity primarily reflected perceptual representations. In the explicit condition, priming increased $c$ relative to Experiment 2, but did not increase $a$. Yonelinas's (2002) analysis indicates that the increase in $c$ can be mediated only conceptually. However, the conceptual impact of the prime did not significantly increase parameter $a$ in the explicit condition relative to Experiment 2 . That favors the view that $a$ was not supported by conceptual representations and must therefore have been supported primarily by perceptual representations.

This proposal receives some support from evidence that conceptual representations are more supportive of controlled than of automatic bases of recognition (Yonelinas, 2002, p. 479). It is also consistent with Long and Prat's (2002) finding that the contribution of recollection to text recognition was greater for readers who had expert knowledge about the theme of the text than for novices, but that the contribution of familiarity was equivalent in the two groups. Relative to novices, experts may be assumed to form richer conceptual text representations of studied material in the form of more elaborated propositional and situational networks. Therefore, the absence of an expert-novice familiarity difference suggests that familiarity was not supported by conceptual representations. The only alternative is that it was supported by perceptual representations.

The modest but significant automatic influences in the motive condition (Experiment 3 ) and control condition (Experiments 2 and 3 ) merit consideration. These effects may reflect the impact of low-level word associations in comprehension. There is evidence that both the intended and unintended associated meanings of the words of a discourse are transiently activated during comprehen- 
sion (Keefe \& McDaniel, 1993; Swinney, 1979; Till, Mross, \& Kintsch, 1988). Activated concepts have at least a brief opportunity to influence the construction of one or more levels of text representation (Kintsch, 1988), and, as a result may become a weak part of that representation (McKoon \& Ratcliff, 1986). To the extent that activation converged on the target word during the reading of the two-sentence text in Phase 1, an automatic influence might result. We consider this hypothesis to be worthy of future scrutiny.

\section{GENERAL DISCUSSION}

In this study, the extended process-dissociation procedure (Buchner et al., 1995) was used to evaluate the controlled and automatic contributions to the retrieval of elaborative and bridging text inferences and corresponding explicit ideas. The results were consistent across three experiments. Both the controlled and automatic influences were greater for explicit targets than for inference targets. Inference recognition received appreciable controlled support but weak or negligible automatic support. These results were interpreted to diagnose differences in the LTM representation of explicit and implicit text ideas. This conclusion, and the ones described next, rest, of course, on the validity of the central assumption that text retrieval is supported by an automatic process of familiarity and a controlled process of recollection. Justification for this assumption was presented in the introduction.

Greater controlled support for explicit than for implicit recognition was interpreted to signal differences in the conceptual representations of the two. This is because the controlled contribution is exclusively associated with conceptual processing (Jacoby, 1991; Yonelinas, 2002). The priming manipulation of Experiment 3 bore on this proposal. Relative to Experiment 2, the only appreciable impact of priming the target word was to increase the controlled parameter of the explicit condition. If the conceptual representations of the explicit and implicit text ideas had been equivalent, priming ought to have affected their controlled support to a similar extent.

The minimal automatic basis of inference recognition suggests that perceptual representations of the inferential concepts were meagre or absent. This conclusion is congruent with the nonappearance of the inference words in the stimulus texts. The conclusion stems in part from the association of automatic support for recognition with both conceptual and perceptual processes (Yonelinas, 2002). It also receives support from indirect evidence in Experiment 3 that automatic contributions to recognition were mainly supported by perceptual representations. In this event, the weak or nonexistent contribution of familiarity to the recognition of inferences would suggest that the perceptual representations of these inferences were sparse. Conversely, the substantial automatic influences in the explicit conditions would suggest that perceptual representations contributed to the recognition of the explicit targets.
We interpret the main patterns of the results to indicate that explicit ideas and bridging inferences are encoded asymmetrically in the surface, textbase, and situation representations of text. In particular, only explicit ideas ought to be robustly encoded in the surface representation (Schmalhofer et al., 2002). As discussed throughout, this proposal is consistent with the outcome that target recognition received appreciable automatic support in the explicit condition.

Differences between the conceptual representations of explicit and implicit text ideas might reside in the propositional textbase or the situation model, both of which capture aspects of text gist. Existing evidence in this realm tends to favor the textbase as the locus of this difference. As discussed earlier, an implicit textbase concept might be expressed indeterminately or in a form corresponding to a variety of related words (McKoon \& Ratcliff, 1986). Explicit text concepts, in contrast, may be reasonably posited to be directly instantiated in the textbase (e.g., Kintsch, 1988; Schmalhofer et al., 2002). Test probes using the original wording would consequently have a retrieval advantage over those with synonym substitutions (Hayes-Roth \& Hayes-Roth, 1977; Kintsch, Welsch, Schmalhofer, \& Zimny, 1990). Conversely, theory (Schmalhofer et al., 2002; Zwaan, Magliano, \& Graesser, 1995) and data (e.g., Rinck, Haehnel, \& Becker, 2001; Suh \& Trabasso, 1993; Wilson, Rinck, McNamara, Bower, \& Morrow, 1993) converge on the conclusion that situation models capture goal, space, time, and other text inferences, particularly ones that conceptually bridge text ideas. This argues against the proposition that differences between the conceptual representation of explicit and implicit text ideas reside in the situation model. A more definitive resolution of this issue might result from combining the present techniques with methods for distinguishing the representational levels (Kintsch et al., 1990).

Consistent with this multilevel analysis is the finding, discussed earlier, that people's text-relevant knowledge augments the recollective component of recognition, but not familiarity (Long \& Prat, 2002). Long and Prat favored the mediation of this effect by gist representations (either textbase or situational) over surface mechanisms. Likewise, in a study of bridging processes, FincherKiefer (1995) reported that recognition time was longer for bridging-inference words that also appeared explicitly elsewhere in a message than for other explicit words that did not capture a bridginginference. She interpreted this outcome as an interference effect, attributing it to the posited presence of the bridging inference concept in (1) the surface representation, which would promote a "yes" response in recognition; and (2) the sort of gist representation that results from inference computation, which would promote a "no" response.

\section{Bridging and Elaborative Text Inferences}

For two reasons, we do not propose that the measured differences between explicit ideas and bridging infer- 
ences challenge the relatively privileged status of bridging inferences in the representation of text. First, the superiority of explicit ideas to bridging-inference concepts in certain recognition tasks (e.g., Fincher-Kiefer, 1995; Singer, 1980) has been treated as a dissociation from answer time and inference-naming time measures rather than as evidence for the noncomputation of the bridging inferences. Such dissociations have the capacity to clarify the contributing information processes. Second, direct comparisons between elaborative and bridging inferences indicate that bridging inferences are more robustly encoded in the message representation (Potts et al., 1988; Singer, 1980; Singer \& Ferreira, 1983). In that regard, one might wonder why the process-dissociation profiles of elaborative inferences (Experiment 1) and bridging inferences (Experiments 2 and 3 ) were qualitatively similar. However, a conclusive comparison between these inference classes would require their examination with reference to similar materials in a single experiment.

There is extensive evidence that the superficial processing of a stimulus, by manipulations such as divided attention (Jacoby et al., 1992; Jacoby et al., 1993) and shallow semantic processing (Dehn \& Engelkamp, 1997; Komatsu et al., 1995; Toth, 1996), yields process-dissociation profiles of a greatly diminished controlled contribution plus a relatively intact automatic contribution. This might raise the question of why, in contrast, the retrieval of the present bridging and elaborative inferences was predominantly controlled. However, text inferences are not aptly characterized in terms of a reduction of semantic processing. First, inferential concepts are not physically present during encoding, whereas superficially processed stimuli are. The physical appearance of a superficially analyzed stimulus affords the application of perceptual processes, which contribute significantly to the automatic support of retrieval (Jacoby, 1991; Mandler, 1980). Second and conversely, the only basis for the judgment that an inferential concept appeared in a text is the semantic derivation of the concept from the explicit text ideas. This derivation likely represents the reader's active involvement in the generation of the inference (Anderson \& Bower, 1972; Duffy et al., 1990). Superficially processed stimuli, in contrast, are characterized by a curtailment of semantic processing. As such, there is little similarity between the encoding of bridging inferences and the superficial processing of a stimulus. These proposed differences are emphatically supported by existing data. To cite two extreme examples, retrieval of the present inferences was based on an automatic contribution at or near zero, whereas the retrieval of stimuli processed under divided attention (Jacoby et al., 1993, Table 2) was based on a controlled contribution of zero.

\section{Alternative Analyses}

The central assumptions of the present analysis are not universally held. According to competing one-process memory models, for example, recognition probes are evaluated with reference to a single global index of mem- ory strength. One-process theories have the merits of accommodating a wide variety of memory phenomena (e.g., the SAM model of Gillund \& Shiffrin, 1984) and of being computationally instantiated (Gillund \& Shiffrin, 1984; Hintzman, 1988; Murdock, 1992). Indeed, Ratcliff, van Zandt, and McKoon (1995) simulated list length and study time effects derived from the process-dissociation procedure in terms of the single process of the SAM model. However, Yonelinas (2002) noted that one-process models often involve two components, such as a contribution to recognition of a controlled recall process (Gillund \& Shiffrin, 1984; Hintzman \& Curran, 1994) or distinct item and association memory information. We conclude that the dual-process analysis of the present phenomena is warranted in the context of existing theory as well as empirical demonstrations (Caldwell \& Masson, 2001; Long \& Prat, 2002).

The proposal that multiple representations (verbatim and gist) support retrieval (Clark \& Gronlund, 1996) presents a different challenge to the dual-process analysis. The dual-representation "conjoint recognition" model (Brainerd et al., 1999) highlights constructs that are ostensively relevant to making recognition judgments about text inferences. These include (1) recognition based on the perceptual processing of an implied concept (generally denied in our analysis) and (2) successful retrieval resulting in rejection rather than false recognition of the related distractor (because the participant notices the difference between the probe and the antecedent). There are reasons that these factors may not have affected our analysis. First, the retrieval of complex implied sentences is unlikely to be based on the processing of their surface forms (Brainerd et al., 1999, p. 165). Second, Clark and Gronlund (1996) identified factors that prevent participants' rejection of related distractors on the basis of successful retrieval of the distractor (e.g., the related distractor spaniel reminding the participant of the list element collie). Nevertheless, conjoint recognition is an intriguing hypothesis that has the capacity to be fruitfully compared with the dual-process model in the domain of text retrieval.

In conclusion, the present study represents an initial application of process dissociation to the examination of the representation of explicit and implicit text ideas. Three experiments yielded stable results that meshed sensibly with prior findings. This offers reasonable assurance that process dissociation will offer further insights concerning the comprehension and representation of text and discourse.

\section{REFERENCES}

ANDERSON, J. R., \& BOWER, G. H. (1972). Configural properties in sentence memory. Journal of Verbal Learning \& Verbal Behavior, 11, 594-605.

BATCHELdER, W. H., \& RiEFER, D. M. (1990). Multinomial processing models of source monitoring. Psychological Review, 97, 548-564.

BlaCK, J. B., \& BERN, H. (1981). Causal inference and memory for events in narratives. Journal of Verbal Learning \& Verbal Behavior, 20, 267-275. 
Bloom, C. P., Fletcher, C. R., van den Broek, P., Reitz, L., \& SHAPIRO, B. P. (1990). An on-line assessment of causal reasoning during comprehension. Memory \& Cognition, 18, 65-71.

Brainerd, C. J., Reyna, V. F., \& Mojardin, A. H. (1999). Conjoint recognition. Psychological Review, 106, 160-179.

Bransford, J. D., \& Franks, J. J. (1971). The abstraction of linguistic ideas. Cognitive Psychology, 2, 331-350.

Buchner, A., Erdfelder, E., \& Vaterrodt-Plunnecke, B. (1995). Toward unbiased measurement of conscious and unconscious memory processes within the process dissociation framework. Journal of Experimental Psychology: General, 124, 137-160.

Caldwell, J. I., \& Masson, M. E. J. (2001). Conscious and unconscious influences of memory for object location. Memory \& Cognition, 29, 285-295.

Clark, S. E., \& Gronlund, S. D. (1996). Global matching models of recognition memory: How the models match the data. Psychonomic Bulletin \& Review, 3, 37-60.

CoOk, A. E., Limber, J. E., \& O'Brien, E. J. (2001). Situation-based context and the availability of predictive inferences. Journal of Memory \& Language, 44, 220-234.

Corbett, A. T., \& Dosher, B. A. (1978). Instrument inferences in sentence encoding. Journal of Verbal Learning \& Verbal Behavior, 17, 479-492.

DEESE, J. (1959). On the prediction of occurrence of particular verbal intrusions in immediate recall. Journal of Experimental Psychology, $58,17-22$.

Denn, D. M., \& Engelkamp, J. (1997). Process dissociation procedure: Double dissociations following divided attention and speeded responding. Quarterly Journal of Experimental Psychology, 50A, 318 336.

DOPKINS, S., KLIN, C., \& MYERS, J. L. (1993). Accessibility of information about goals during the processing of narrative texts. Journal of Experimental Psychology: Learning, Memory, \& Cognition, 19 , 70-80.

DufFy, S. A., ShInJo, M., \& MYers, J. L. (1990). The effect of encoding task on memory for sentence pairs varying in causal relatedness. Journal of Verbal Learning \& Verbal Behavior, 29, 27-42.

Fillmore, C. J. (1968). The case for case. In E. Bach \& R. T. Harms (Eds.), Universals of linguistic theory (pp. 1-90). New York: Holt, Rinehart \& Winston.

FinCHER-KiEfER, R. (1995). Relative inhibition following the encoding of bridging and predictive inferences. Journal of Experimental Psychology: Learning, Memory, \& Cognition, 21, 981-995.

Gardiner, J. M., GaWlik, B., \& Richardson-Klavehn, A. (1994). Maintenance rehearsal affects knowing, not remembering; elaborative rehearsal affects remembering, not knowing. Psychonomic Bulletin \& Review, 1, 107-110.

GARDINER, J. M., \& JAVA, R. L. (1991). Forgetting in recognition memory with and without recollective experience. Memory \& Cognition, $19,617-623$

Gillund, G., \& Shiffrin, R. M. (1984). A retrieval model for both recognition and recall. Psychological Review, 91, 1-67.

Graf, P., \& Komatsu, S. (1994). Process dissociation procedure: Handle with caution! European Journal of Cognitive Psychology, 6, 113-129.

HASHER, L., \& GRIFFIN, M. (1978). Reconstructive and reproductive processes in memory. Journal of Experimental Psychology: Human Learning \& Memory, 4, 318-330.

HAYES-RoTh, B., \& HAYES-ROTH, F. (1977). The prominence of lexical information in memory representations of meaning. Journal of Verbal Learning \& Verbal Behavior, 16, 119-136.

Hintzman, D. L. (1988). Judgments of frequency and recognition memory in a multiple-trace memory model. Psychological Review, 95, 528-551.

Hintzman, D. L., \& Caulton, D. A. (1997). Recognition memory and modality judgments: A comparison of retrieval dynamics. Journal of Memory \& Language, 37, 1-23.

HintzMan, D. L., \& CURRan, T. (1994). Retrieyal dynamics of recognition and frequency judgments: Evidence for separate processes of familiarity and recall. Journal of Memory \& Language, 33, 1-18.

Hu, X., \& BATCHELDER, W. H. (1994). The statistical analysis of gen- eral processing tree models with the EM algorithm. Psychometrika, $\mathbf{5 9}, 21-47$

Hu, X., \& PHILliPs, G. A. (1999). GPT.EXE: A powerful tool for the visualization and analysis of general processing tree models. Behavior Research Methods, Instruments, \& Computers, 31, 220-234.

JACOBY, L. L. (1991). A process dissociation framework: Separating automatic from intentional uses of memory. Journal of Memory \& Language, 30, 513-541.

JACORY, J. L., LINDSAY, D. S., \& ToTH, J. P. (1992). Unconscious influences revealed. American Psychologist, 47, 802-809.

JACOBY, L. L., TOTH, J. P., \& Yonelinas, A. P. (1993). Separating conscious and unconscious influences of memory: Measuring recollection. Journal of Experimental Psychology: General, 122, 139-154.

Johnson, M. K., Bransford, J. D., \& Solomon, S. K. (1973). Memory for tacit implications of sentences. Journal of Experimental Psychology, 98, 203-205.

JoORdens, S., \& MERikLe, P. M. (1993). Independence or redundancy. Two models of conscious and unconscious influences. Journal of Experimental Psychology: General, 122, 462-467.

KEEFE, D. E., \& MCDANIEL, M. A. (1993). The time course and durability of predictive inferences. Journal of Memory \& Language, 32 , 446-463.

Keenan, J. M., Baillet, S. D., \& Brown, P. (1984). The effects of causal cohesion on comprehension and memory. Journal of Verbal Learning \& Verbal Behavior, 23, 115-126.

KINTSCH, W. (1988). The role of knowledge in discourse comprehension: A construction-integration model. Psychological Review, 95, 163-182.

Kintsch, W., Welsch, D., Schmalhofer, F., \& Zimny, S. (1990). Sentence memory: A theoretical analysis. Journal of Memory \& Language, 29, 133-159.

KLIN, C. M. (1995). Causal inferences in reading: From immediate activation to long term memory. Journal of Experimental Psychology: Learning, Memory, \& Cognition, 21, 1483-1494.

KiIn, C. M., Guzman, A. E., \& Levine, W. H. (1999). Prevalence and persistence of predictive inferences. Journal of Memory \& Language, 40, 593-604.

Komatsu, S., Graf, P., \& Utrl, B. (1995). Process dissociation procedure: Core assumptions fail, sometimes. European Journal of Cognitive Psychology, 7, 19-40.

Levy, B. H., Campsall, J., Browne, J., CoOper, D., Waterhouse, C., \& WILSON, C. (1995). Reading fluency: Episodic integration across tests. Journal of Experimental Psychology: Learning, Memory, \& Cognition, 21, 1169-1185.

LONG, D. L., \& PRAT, C. S. (2002). Memory for Star Trek: The role of prior knowledge in recognition revisited. Journal of Experimental Psychology: Learning, Memory, \& Cognition, 28, 1073-1082.

Lucas, M. M., Tanenhaus, M. K., \& Carlson, G. N. (1990). Levels of representation in the interpretation of anaphoric reference and instrument inference. Memory \& Cognition, 18, 611-631.

MANDLER, G. (1980). Recognizing: The judgment of previous occurrence. Psychological Review, 87, 252-271.

Masson, M. E. (1993). Episodically enhanced comprehension fluency. Canadian Journal of Experimental Psychology, 47, 428-465.

MCDaniel, M., SChmalhofer, F., \& KeEFE, D. E. (2001). What is minimal about predictive inferences. Psychonomic Bulletin \& Review, 8, 840-846.

MCKoon, G., \& RatclifF, R. (1986). Inferences about predictable events. Journal of Experimental Psychology: Learning, Memory, \& Cognition, 12, 82-91

MCKoon, G., \& RATCLIFF, R. (1988). Contextually relevant aspects of meaning. Journal of Experimental Psychology: Learning, Memory, \& Cognition, 14, 331-343.

Moravcsik, J. E., \& KintsCH, W. (1993). Writing quality, reading skills, and domain knowledge as factors in text comprehension. Canadian Journal of Experimental Psychology, 47, 360-374.

MURDOCK, B. B. (1992). Item and associative information in a distributed memory model. Journal of Mathematical Psychology, 36, 68-99.

Myers, J. L., ShINJo, M., \& DufFy, S. A. (1987). Degree of causal relatedness and memory. Journal of Verbal Learning \& Verbal Behavior, 26, 453-465. 
O'Brien, E. J., Shank, D. M., Myers, J. L., \& Rayner, K. (1988). Elaborative inferences during reading: Do they occur on-line? Journal of Experimental Psychology: Learning, Memory, \& Cognition, 14, $410-420$

PerfetTI, C. A. (1989). There are generalized abilities and one of them is reading. In L. Resnick (Ed.), Knowing and learning: Issues for a cognitive science of instruction (pp. 307-335). Hillsdale, NJ: Erlbaum.

Potts, G. R., Keenan, J. M., \& Golding, J. M. (1988). Assessing the occurrence of elaborative inferences: Lexical decision versus naming. Journal of Memory \& Language, 27, 399-415.

RatCliff, R., van ZANDT, T., \& MCKOON, G. (1995). Process dissociation, single process theories, and recognition memory. Journal of Experimental Psychology: General, 124, 352-374.

REDER, L. M. (1982). Plausibility judgements versus fact retrieval: Alternative strategies for sentence verification. Psychological Review, 89, 250-280.

REDER, L. M. (1987). Strategy-selection in question answering. Cognitive Psychology, 19, 90-134.

RiNCK, M., HAEHNEL, A., \& BECKER, G. (2001). Using temporal information to construct, update, and retrieve situation models of narratives. Journal of Experimental Psychology: Learning, Memory, \& Cognition, 27, 67-80.

Roediger, H. L., III, \& McDermott, K. B. (1995). Creating false memories: Remembering words not presented in lists. Journal of Experimental Psychology: Learning, Memory, \& Cognition, 21, 803-814.

SCHMalhofer, F., \& Glavanov, D. (1986). Three components of understanding a programmer's manual: Verbatim, propositional, and situation representations. Journal of Memory \& Language, 25, 279-294.

Schmalhofer, F., McDaniel, M. A., \& KeEfe, D. E. (2002). A unified model for predictive and bridging inferences. Discourse Processes, 33, 105-132.

SINGER, M. (1980). The role of case-filling inferences in the coherence of brief passages. Discourse Processes, 3, 185-201.

SINGER, M. (1981). Verifying the assertions and implications of language. Journal of Verbal Learning \& Verbal Behavior, 20, 46-60.

SinGER, M. (1994). Discourse inference processes. In M. A. Gernsbacher (Ed.), Handbook of psycholinguistics (pp. 479-515). San Diego: Academic Press.

Singer, M., \& Ferreira, F. (1983). Inferring consequences in story comprehension. Journal of Verbal Learning \& Verbal Behavior, 22, 437-448.

Singer, M., \& Halldorson, M. (1996). Constructing and validating motive bridging inferences. Cognitive Psychology, 30, 1-38.

Singer, M., Halldorson, M., Lear, J. C., \& Andrusiak, P. (1992). Validation of causal bridging inferences. Journal of Memory \& Language, 31, 507-524.

SINGER, M., \& KINTSCH, W. (2001). Text retrieval: A theoretical exploration. Discourse Processes, 31, 27-59.

SlameCKa, N. J., \& Graf, P. (1978). The generation effect: Delineation of a phenomenon. Journal of Experimental Psychology: Human Learning \& Memory, 4, 592-604.

SuH, S., \& Trabasso, T. (1993). Inferences during reading: Converging evidence from discourse analysis, talk-aloud protocols and recognition priming. Journal of Memory \& Language, 32, 279-300.

SWINNEY, D. A. (1979). Lexical access during sentence comprehension:
(Re)consideration of context effects. Journal of Verbal Learning $\&$ Verbal Behavior, 18, 645-659.

Till, R. E., Mross, E. F., \& KinTSCh, W. (1988). Time course of priming for associate and inference words in a discourse context. Memory \& Cognition, 16, 283-298.

Tотн, J. P. (1996). Conceptual automaticity in recognition memory: Levels-of-processing effects on familiarity. Canadian Journal of Experimental Psychology, 50, 123-138.

ToTH, J. P., REINGOLD, E. M., \& JACOBY, L. L. (1994). A response to Graf and Komatsu's critique of the process dissociation procedure: When is caution necessary? European Journal of Cognitive Psychology, 7, 113-130.

Tulving, E. (1985). Memory and consciousness. Canadian Psychology, 26, 1-12.

UNDERWOOD, B. J. (1965). False recognition produced by implicit verbal responses. Journal of Experimental Psychology, 70, 122-129.

van DiJK, T. A., \& KINTsch, W. (1983). Strategies of discourse comprehension. New York: Academic Press.

Wilson, S. G., Rinck, M., MCNamara, T. P., Bower, G. H., \& MorROW, D. G. (1993). Mental models and narrative comprehension: Some qualifications. Journal of Memory \& Language, 32, 141-154.

Yonelinas, A. P. (200I). Consciousness, control, and confidence: The 3 Cs of recognition memory. Journal of Experimental Psychology: General, 130, 361-379.

YONELINAS, A. P. (2002). The nature of recollection and familiarity: A review of 30 years of research. Journal of Memory \& Language, 46, 441-517.

ZwaAn, R. A., Magliano, J. P., \& Graesser, A. C. (1995). Dimensions of situation model construction in narrative comprehension. Journal of Experimental Psychology: Learning, Memory, \& Cognition, 21, 386-397.

\section{NOTES}

1. If controlled and automatic processes are assumed to be independent, then the conditional probability can be interpreted as an unconditional probability (for details, see Buchner et al., 1995).

2. The higher the value of $G^{2}$, the worse the fit of the model to the data. $G^{2}$ for a submodel constrained by a null hypothesis will always be greater than or equal to $G^{2}$ for the full model because the former has fewer free parameters. The question is whether $G^{2}$ for the submodel is significantly greater than that for the full model (i.e., does the constraint imposed by the null hypothesis significantly reduce the fit of the full model?). If so, the null hypothesis is rejected.

$G^{2}$ for the full model in Experiment 1 (Appendix B) was 3.32. The $G^{2}$ values for the full models in Experiments 2 and 3 (Appendix D) were both 0 .

3. Given that familiarity is supported by both conceptual and perceptual representations and there were conceptual representations of elaborative inferences (as indicated by a significant recollection component), the absence of a familiarity component suggests that conceptual representations of elaborative inferences are robust enough to support recollection but not familiarity. This is consistent with evidence that conceptual representations are more supportive of recollection than of familiarity (Yonelinas, 2002). 
APPENDIX A

Significant Effects in Experiment 1 Analysis of Variance

\begin{tabular}{cl}
\hline \multicolumn{1}{c}{ Effect } & \multicolumn{1}{c}{ Statistics } \\
\hline Instruction (I) & $F_{1}(1,152)=118.30, M S_{\mathrm{e}}=.10$ \\
& $F_{2}(1,46)=300.49, M S_{\mathrm{e}}=120$ \\
Relation (R) & $F_{1}(2,304)=183.79, M S_{\mathrm{e}}=.02$ \\
& $F_{2}(2,92)=109.01, M S_{\mathrm{e}}=117$ \\
$\mathrm{I} \times \mathrm{R}$ & $F_{1}(2,304)=70.55, M S_{\mathrm{e}}=.02$ \\
& $F_{2}(2,92)=53.46, M S_{\mathrm{e}}=91$ \\
$\mathrm{I} \times$ probability (P) & $F_{1}(1,152)=18.81, M S_{\mathrm{e}}=.01$ \\
& $F_{2}(1,46)=6.84, M S_{\mathrm{e}}=120$ \\
$\mathrm{R} \times \mathrm{P}$ & $F_{1}(2,304)=10.73, M S_{\mathrm{e}}=.02$ \\
& $F_{2}(2,92)=5.35, M S_{\mathrm{e}}=116$ \\
\hline
\end{tabular}

Note-Statistics are reported only for those effects significant both in the participants-random and items-random ANOVAs.

\section{APPENDIX B}

Processing Tree Model for OLD/New Judgments in Experiment 1

\begin{tabular}{|c|c|c|c|}
\hline ng Path & esp & Processing Path & ise \\
\hline Inclusion & & Exclusion & \\
\hline $\begin{array}{l}\text { Explicit High } \\
\qquad\left(c_{\mathrm{EH}}\right) \\
\quad\left(1-c_{\mathrm{EH}}\right)\left(a_{\mathrm{EH}}\right) \\
\quad\left(1-c_{\mathrm{EH}}\right)\left(1-a_{\mathrm{EH}}\right)\left(g_{\mathrm{iH}}\right) \\
\quad\left(1-c_{\mathrm{EH}}\right)\left(1-a_{\mathrm{EH}}\right)\left(1-g_{\mathrm{iH}}\right)\end{array}$ & $\begin{array}{l}\text { "old" } \\
\text { "old" } \\
\text { "old" } \\
\text { "new" }\end{array}$ & $\begin{array}{l}\text { Explicit High } \\
\qquad \begin{array}{l}\left(c_{\mathrm{EH}}\right) \\
\quad\left(1-c_{\mathrm{EH}}\right)\left(a_{\mathrm{EH}}\right) \\
\quad\left(1-c_{\mathrm{EH}}\right)\left(1-a_{\mathrm{EH}}\right)\left(g_{\mathrm{eH}}\right) \\
\quad\left(1-c_{\mathrm{EH}}\right)\left(1-a_{\mathrm{EH}}\right)\left(1-g_{\mathrm{eH}}\right)\end{array}\end{array}$ & $\begin{array}{l}\text { "new" } \\
\text { "old" } \\
\text { "old" } \\
\text { "new" }\end{array}$ \\
\hline $\begin{array}{l}\text { Implicit High } \\
\qquad\left(c_{\mathrm{IH}}\right) \\
\quad\left(1-c_{\mathrm{IH}}\right)\left(a_{\mathrm{IH}}\right) \\
\quad\left(1-c_{\mathrm{IH}}\right)\left(1-a_{\mathrm{IH}}\right)\left(g_{\mathrm{HH}}\right) \\
\quad\left(1-c_{\mathrm{IH}}\right)\left(1-a_{\mathrm{IH}}\right)\left(1-g_{\mathrm{iH}}\right)\end{array}$ & $\begin{array}{l}\text { "old" } \\
\text { "old" } \\
\text { "old" } \\
\text { "new" }\end{array}$ & $\begin{array}{l}\text { Implicit High } \\
\qquad \begin{array}{l}\left(c_{\mathrm{IH}}\right) \\
\quad\left(1-c_{\mathrm{IH}}\right)\left(a_{\mathrm{IH}}\right) \\
\left(1-c_{\mathrm{IH}}\right)\left(1-a_{\mathrm{IH}}\right)\left(g_{\mathrm{eH}}\right) \\
\left(1-c_{\mathrm{IH}}\right)\left(1-a_{\mathrm{IH}}\right)\left(1-g_{\mathrm{eH}}\right)\end{array}\end{array}$ & $\begin{array}{l}\text { "new" } \\
\text { "old" } \\
\text { "old" } \\
\text { "new" }\end{array}$ \\
\hline $\begin{array}{l}\text { Absent (High) } \\
\left(g_{\mathrm{iH}}\right) \\
\left(1-g_{\mathrm{iH}}\right)\end{array}$ & $\begin{array}{l}\text { "old" } \\
\text { "new" }\end{array}$ & $\begin{array}{l}\text { Absent (High) } \\
\left(g_{\mathrm{eH}}\right) \\
\left(1-g_{\mathrm{eH}}\right)\end{array}$ & \\
\hline $\begin{array}{l}\text { Explicit Low } \\
\quad\left(c_{\mathrm{EL}}\right) \\
\quad\left(1-c_{\mathrm{EL}}\right)\left(a_{\mathrm{EL}}\right) \\
\quad\left(1-c_{\mathrm{EL}}\right)\left(1-a_{\mathrm{EL}}\right)\left(g_{\mathrm{iL}}\right) \\
\quad\left(1-c_{\mathrm{EL}}\right)\left(1-a_{\mathrm{EL}}\right)\left(1-g_{\mathrm{iL}}\right)\end{array}$ & $\begin{array}{l}\text { "old" } \\
\text { "old" } \\
\text { "old" } \\
\text { "new" }\end{array}$ & $\begin{array}{l}\text { Explicit Low } \\
\qquad\left(c_{\mathrm{EL}}\right) \\
\quad\left(1-c_{\mathrm{EL}}\right)\left(a_{\mathrm{EL}}\right) \\
\quad\left(1-c_{\mathrm{EL}}\right)\left(1-a_{\mathrm{EL}}\right)\left(g_{\mathrm{eL}}\right) \\
\quad\left(1-c_{\mathrm{EL}}\right)\left(1-a_{\mathrm{EL}}\right)\left(1-g_{\mathrm{eL}}\right)\end{array}$ & $\begin{array}{l}\text { "new" } \\
\text { "old" } \\
\text { "old" } \\
\text { "new" }\end{array}$ \\
\hline $\begin{array}{l}\text { mplicit Low } \\
\left(c_{\mathrm{IL}}\right) \\
\left(1-c_{\mathrm{IL}}\right)\left(a_{\mathrm{IL}}\right) \\
\left(1-c_{\mathrm{IL}}\right)\left(1-a_{\mathrm{IL}}\right)\left(g_{\mathrm{iL}}\right) \\
\left(1-c_{\mathrm{IL}}\right)\left(1-a_{\mathrm{IL}}\right)\left(1-g_{\mathrm{iL}}\right)\end{array}$ & $\begin{array}{l}\text { "old" } \\
\text { "old" } \\
\text { "old" } \\
\text { "new" }\end{array}$ & $\begin{array}{l}\text { Implicit Low } \\
\quad\left(c_{\mathrm{IL}}\right) \\
\quad\left(1-c_{\mathrm{IL}}\right)\left(a_{\mathrm{IL}}\right) \\
\quad\left(1-c_{\mathrm{IL}}\right)\left(1-a_{\mathrm{IL}}\right)\left(g_{\mathrm{eL}}\right) \\
\quad\left(1-c_{\mathrm{IL}}\right)\left(1-a_{\mathrm{IL}}\right)\left(1-g_{\mathrm{eL}}\right)\end{array}$ & $\begin{array}{l}\text { "new" } \\
\text { "old" } \\
\text { "old" } \\
\text { "new" }\end{array}$ \\
\hline $\begin{array}{l}\text { Absent (Low) } \\
\quad\left(g_{i L}\right) \\
\left(1-g_{i L}\right)\end{array}$ & $\begin{array}{l}\text { "old" } \\
\text { "new" }\end{array}$ & $\begin{array}{l}\text { Absent (Low) } \\
\left(g_{\mathrm{eL}}\right) \\
\left(1-g_{\mathrm{eL}}\right)\end{array}$ & $\begin{array}{l}\text { "old" } \\
\text { "new" }\end{array}$ \\
\hline
\end{tabular}

Note $-c=$ the probability that a target word (from the subscripted condition) will be recollected as being from Phase $1 ; a=$ the probability that the familiarity of a target word (from the subscripted condition) will exceed the thresh"old" for responding "old" given that it is not recollected as being from Phase $1 ; g_{\mathrm{j}}$ and $g_{\mathrm{e}}=$ the probabilities of guessing "old" in the inclusion and exclusion conditions, respectively, given that a target word is not recollected as being from Phase 1 and its familiarity does not exceed the response thresh"old". 


\begin{tabular}{|c|c|c|c|c|c|}
\hline \multirow[b]{3}{*}{ Experiment } & \multirow[b]{3}{*}{ Condition } & $\begin{array}{l}\text { DIX } \\
\text { Reco } \\
\text { ents }\end{array}$ & ion & 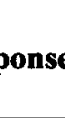 & \\
\hline & & \multicolumn{2}{|c|}{ Inclusion } & \multicolumn{2}{|c|}{ Exclusion } \\
\hline & & Old & New & Old & New \\
\hline 1 & $\begin{array}{l}\text { Explicit High } \\
\text { Implicit High } \\
\text { Absent High } \\
\text { Explicit Low } \\
\text { Implicit Low } \\
\text { Absent Low }\end{array}$ & $\begin{array}{r}262 \\
157 \\
209 \\
250 \\
86 \\
175\end{array}$ & $\begin{array}{l}200 \\
305 \\
715 \\
212 \\
376 \\
749\end{array}$ & $\begin{array}{l}70 \\
46 \\
91 \\
85 \\
30 \\
86\end{array}$ & $\begin{array}{l}392 \\
416 \\
833 \\
377 \\
432 \\
838\end{array}$ \\
\hline 2 & $\begin{array}{l}\text { Explicit } \\
\text { Motive } \\
\text { Control } \\
\text { Absent }\end{array}$ & $\begin{array}{r}154 \\
92 \\
66 \\
43\end{array}$ & $\begin{array}{l}118 \\
180 \\
206 \\
229\end{array}$ & $\begin{array}{l}34 \\
16 \\
27 \\
14\end{array}$ & $\begin{array}{l}238 \\
256 \\
245 \\
258\end{array}$ \\
\hline 3 & $\begin{array}{l}\text { Explicit } \\
\text { Motive } \\
\text { Control } \\
\text { Absent }\end{array}$ & $\begin{array}{r}180 \\
99 \\
74 \\
45\end{array}$ & $\begin{array}{r}72 \\
153 \\
178 \\
207\end{array}$ & $\begin{array}{l}33 \\
24 \\
27 \\
16\end{array}$ & $\begin{array}{l}223 \\
232 \\
229 \\
240\end{array}$ \\
\hline
\end{tabular}

\section{APPENDIX D}

Processing Tree Model for OLD/New Judgments

in Experiments 2 and 3

\begin{tabular}{|c|c|c|c|}
\hline Processing Path & Response & Processing Path & Response \\
\hline Inclusion & & Exclusion & \\
\hline $\begin{array}{l}\text { Explicit } \\
\qquad\left(c_{\mathrm{E}}\right) \\
\quad\left(1-c_{\mathrm{E}}\right)\left(a_{\mathrm{E}}\right) \\
\quad\left(1-c_{\mathrm{E}}\right)\left(1-a_{\mathrm{E}}\right)\left(g_{\mathrm{i}}\right) \\
\quad\left(1-c_{\mathrm{E}}\right)\left(1-a_{\mathrm{E}}\right)\left(1-g_{\mathrm{i}}\right)\end{array}$ & $\begin{array}{l}\text { "old" } \\
\text { "old" } \\
\text { "old" } \\
\text { "new" }\end{array}$ & $\begin{array}{l}\text { Explicit } \\
\qquad \begin{array}{l}\left(c_{\mathrm{E}}\right) \\
\quad\left(1-c_{\mathrm{E}}\right)\left(a_{\mathrm{E}}\right) \\
\quad\left(1-c_{\mathrm{E}}\right)\left(1-a_{\mathrm{E}}\right)\left(g_{\mathrm{e}}\right) \\
\quad\left(1-c_{\mathrm{E}}\right)\left(1-a_{\mathrm{E}}\right)\left(1-g_{\mathrm{e}}\right)\end{array}\end{array}$ & $\begin{array}{l}\text { "new" } \\
\text { "old" } \\
\text { "old" } \\
\text { "new" }\end{array}$ \\
\hline $\begin{array}{l}\text { Motive } \\
\qquad \begin{array}{l}\left(c_{M}\right) \\
\quad\left(1-c_{M}\right)\left(a_{M}\right) \\
\quad\left(1-c_{M}\right)\left(1-a_{M}\right)\left(g_{i}\right) \\
\quad\left(1-c_{M}\right)\left(1-a_{M}\right)\left(1-g_{i}\right)\end{array}\end{array}$ & $\begin{array}{l}\text { “old" } \\
\text { "old" } \\
\text { "old" } \\
\text { "new" }\end{array}$ & $\begin{array}{l}\text { Motive } \\
\qquad\left(c_{M}\right) \\
\qquad\left(1-c_{M}\right)\left(a_{M}\right) \\
\quad\left(1-c_{M}\right)\left(1-a_{M}\right)\left(g_{e}\right) \\
\quad\left(1-c_{M}\right)\left(1-a_{M}\right)\left(1-g_{e}\right)\end{array}$ & $\begin{array}{l}\text { "new" } \\
\text { "old" } \\
\text { "old" } \\
\text { "new" }\end{array}$ \\
\hline $\begin{array}{l}\text { Control } \\
\qquad \begin{array}{l}\left(c_{\mathrm{C}}\right) \\
\quad\left(1-c_{\mathrm{C}}\right)\left(a_{\mathrm{C}}\right) \\
\quad\left(1-c_{\mathrm{C}}\right)\left(1-a_{\mathrm{C}}\right)\left(g_{\mathrm{i}}\right) \\
\quad\left(1-c_{\mathrm{C}}\right)\left(1-a_{\mathrm{C}}\right)\left(1-g_{\mathrm{i}}\right)\end{array}\end{array}$ & $\begin{array}{l}\text { "old" } \\
\text { "old" } \\
\text { "old" } \\
\text { "new" }\end{array}$ & $\begin{array}{l}\text { Control } \\
\qquad \begin{array}{l}\left(c_{\mathrm{C}}\right) \\
\quad\left(1-c_{\mathrm{C}}\right)\left(a_{\mathrm{C}}\right) \\
\left(1-c_{\mathrm{C}}\right)\left(1-a_{\mathrm{C}}\right)\left(g_{\mathrm{e}}\right) \\
\left(1-c_{\mathrm{C}}\right)\left(1-a_{\mathrm{C}}\right)\left(1-g_{\mathrm{e}}\right)\end{array}\end{array}$ & $\begin{array}{l}\text { "new" } \\
\text { "old" } \\
\text { "old" } \\
\text { "new" }\end{array}$ \\
\hline $\begin{array}{l}\text { Absent } \\
\quad\left(g_{i}\right) \\
\left(1-g_{i}\right)\end{array}$ & $\begin{array}{l}\text { "old" } \\
\text { "new" }\end{array}$ & $\begin{array}{l}\text { Absent } \\
\qquad\left(g_{\mathrm{e}}\right) \\
\left(1-g_{\mathrm{e}}\right)\end{array}$ & $\begin{array}{l}\text { "old" } \\
\text { "new" }\end{array}$ \\
\hline
\end{tabular}

Note $-c=$ the probability that a target word (from the subscripted condition) will be recollected as being from Phase 1; $a$ = the probability that the familiarity of a target word (from the subscripted condition) will exceed the threshold for responding "old" given that it is not recollected as being from Phase $1 ; g_{\mathrm{i}}$ and $g_{\mathrm{c}}=$ the probabilities of guessing "old" in the inclusion and exclusion conditions, respectively, given that a target word is not recollected as being from Phase 1 and its familiarity does not exceed the response threshold.

(Manuscript received September 4, 2002;

revision accepted for publication February 25, 2004.) 